\title{
Progress in Bioremediation Technology of groundwater contamination
}

\author{
Erxing Guan ${ }^{1, a}$, Deshen Liu, ${ }^{*}$, b \\ ${ }^{1}$ Guilin University of Technology School of environment and Engineering, Guilin University of \\ Technology, Guilin 541004, China \\ aGuan_ex@163.com, ${ }^{\mathrm{b}} 420917694 @ q q . c o m$
}

*Corresponding author: Deshen Liu. Email:420917694@qq.com

Keywords: groundwater pollution; bioremediation; technology application

\begin{abstract}
Recently groundwater in the world is polluted seriously, threating the health of human directly or indirectly.Therefore the remediation of groundwater has raised people's attention. This paper introduces the concept and characteristics of groundwater bioremediation technology,discussing the repair repair mechanism, object repair technology and its technical characteristics. A comprehensive comparative evaluation of above repair technology project investment, operation cost and treatment time, provide the necessary theoretical basis for the implementation of groundwater pollution remediation. Finally, the development direction of groundwater remediation technology is prospected.
\end{abstract}

\section{Introduction}

In recent years, due to a large number of industrial and agricultural waste unreasonable landfill, the leakage accident of pollutants emission underground storage facilities and a variety of organic compounds, heavy metals and radioactive hazardous substances enter groundwater system, the groundwater pollution is becoming more and more serious[1]. The remediation of contaminated groundwater and strengthening groundwater environmental protection has becoming a hotspot of current research at home and abroad. With the rapid development of industrial production, and the resulting industrial waste water, oil spills, excessive pesticide and fertilizer application, etc.,so that contamination of soil and groundwater is becoming more serious, problem in this regard has also been more and more attention.

Environmental biotechnology can be divided into low, medium and high level. Low level refers to the use of natural treatment system for waste treatment technology[2], such as oxidation pond and artificial wetland, it can give full play to the natural biological purification function, low investment and running costs, easy to manage. Medium level is refers to the traditional biological treatment technology, such as activated sludge process and the biological membrane method, and in the context of the new theory and technology of strengthening treatment technology and process, such as biological fluidized bed and bioaugmentation technology etc..High level technology refers to the gene engineering, enzyme engineering and other modern biological technology as the basis for the prevention and control of pollution biotechnology, such as gene engineering bacteria construction, is fast and effective pollution prevention and control provides a new way.

The final conversion product processing pollutants with biological method are non-toxic and stable material,such as carbon dioxide, water, nitrogen and methane etc., it avoids the repeated transfer of pollutants. Therefore, the biological technology is a safe means of completely eliminating pollution [3].

\section{The concept and characteristics of bioremediation technology}

The so-called bioremediation technology is a method of processing technology that refers to the use of natural or special culture of biological (plant, microorganism and protozoa) in controlled environmental conditions will be toxic pollutants into non-toxic materia[4]. Compared with the 
traditional physical and chemical remediation technology,Compared with the traditional technology of physical and chemical remediation, bioremediation has the following advantages:

(1) Bioremediation can work on site, so transportation costs and opportunities for direct human exposure to pollutants will be reduced;

(2) Bioremediation is often carried out through in situ, so that the interference or destruction of the pollution site is minimal;

(3) Bioremediation can put organic compounds into carbon dioxide and water. This treatment had no two pollution, will not cause the transfer of pollutants;

(4) Bioremediation can be used in combination with other processing technology, so that it can process compound pollution;

(5) The degradation process is rapid, low cost, only the traditional physical and chemical remediation 30\% 50\%.

\section{The application of bioremediation of groundwater pollution}

The simplest method of removal of pollutants pumps or discharges the contaminated water to the ground for processing using the water wells or drainage ditches, then re injected into the aquifer. Bioremediation is the use of the natural environment in the growth of microorganisms or adding specific microorganisms, decomposition of pollutants in human engineering to promote conditions, in order to repair the polluted environment. Treatment of contaminated groundwater aquifer is difficult, many methods are still in the exploratory stage, the need to continue to study in the theory field in the future, at the same time in the technical innovation and improvement.

\subsection{Peat biological barrier method}

Peat, also known as peat moss or peat coal, with chelating structure. It can react quickly and ion exchanged with other ions or radicals, which will be transferred to the solid contaminants from the water, to remove pollutants in water .

Xuesong Yi etc.[5] had studied peat adsorption of $\mathrm{Pb}^{2+}$,found that Peat has strong adsorption of $\mathrm{Pb}^{2+}$.In the experiment that peat adsorb the $\mathrm{pb}^{2+}$ maximum capacity is $96.85 \mathrm{mg} / \mathrm{g}$,when the amount of $12 \mathrm{~g} / \mathrm{L}$ of peat, peat's $\mathrm{Pb}^{2+}$ removal rate is $96.2 \%$.

Peat is easy to achieve, but it is inconvenient to use. This is mainly due to natural peat is highly hydrophilic, poor chemical stability, easy contraction and expansion, the adsorption ability is poor, so it should be modified appropriately to improve its chemical properties [6]

\subsection{In situ bioremediation technology}

In situ bioremediation methods of groundwater is by oxygen and nutrients to the aquifer, rely on indigenous microbial to decompose pollutants. Currently on organic pollution of groundwater by in situ bioremediation method mainly including biological injection method, organic clay method, extraction groundwater system and reinjection system combined method.

\subsubsection{Air injection technology to repair}

AS technology is developed on basis of soil gas phase extraction SVE Technology (SVE). In the middle period of the eighties of the 20th century the earliest application in Germany [7], followed by a rapid development to the United States and other countries of Europe. In recent years, AS technology has become the first choice for in situ groundwater treatment technology.

AS technology is sometimes defined as the in situ biological aeration technology, no clear dividing line between the two technology, generally depends on the dominant position of the control factors of in different conditions and operation stage[14]. The main factors including evaporation, desorption and biodegradation of several parts.In general, based on physical volatile in the early treatment, while in the latter dominated by biodegradation of governance.

The technique is commonly used in treatment of underground saturated zone (zone of saturation and capillary saturated zone) of organic pollution, general and SVE technology used in combination, the repair principle is: by injecting air into the ground, at the bottom of the pollution plume formed air barrier, prevent pollution halo further downward diffusion and migration, in under the action of pressure gradient collected underground volatile contaminants, and oxygen as the main means, promote the biodegradation of subsurface contaminants. 
AS technology has the following characteristics:Simple equipment, convenient installation, easy operation;High repair efficiency, short treatment time, generally under repair period for $1 \sim 4 \mathrm{a}[8]$; More suitable for removing the groundwater is difficult to move the pollutant treatment; in-situ repair and to point repair interference small.The limitations of the technique are mainly the following several points:For non volatile pollutants does not apply;By geological conditions, not suitable for use in regions with low permeability or high clay content;Cannot be applied to a confined with stratification by soil and water pollution control.

\subsubsection{Organic clay method}

Organic clay is a method of in situ bioremediation of contaminated groundwater development. It uses the synthetic organic clay effectively remove toxic compounds. Namely cation surface active agent through injection well is injected into the water storage layer, through chemical bond to with negatively charged clay surface, synthetic organic clay minerals, thus forming effective adsorption zone, control of toxic compounds in groundwater migration, the field of microbial, degrading enrichment in the adsorption of organic pollutants so as to eliminate the groundwater organic pollutants.

Used in the technology of surface active agent is composed of synthetic fatty acid derivatives, alkyl sulfonate, alkyl benzene sulfonates, alkyl sulfate organic compounds. Because the hydrophilic and hydrophobic properties of the two molecules, so they tend to congregate in the air - water interface and oil - water interface, can reduce the surface tension, promote emulsification.

\subsubsection{Groundwater extraction system and injection system combination}

This method will be groundwater extraction system and injection system (air or H2O2, nutrients and domesticated microorganism injection) combine to promote the biodegradation of organic pollutants. Smallbeck et al [9] in the study of the California delta showed that using this system for remediation of contaminated environment, biological degradation significantly promoted. This system not only can save processing costs, and shorten the processing time, is undoubtedly an effective method.

\subsection{Off - situ bioremediation}

At present, off-situ bioremediation methods of groundwater is mainly bioreactor.

Processing method of the bioreactor is the groundwater extraction process on the part of bioreactor for processing, the natural formation of a closed cycle. With the conventional wastewater treatment, reactor type used in a variety of ways.

In general, treatment bioreactor has the following process:

(1) The contaminated groundwater extraction to the ground;

(2) Aerobic degradation the groundwater in the ground of bioreactor,The reactor of nutrients and oxygen in the process of operation;

(3) After the treatment of groundwater recharge by infiltration irrigation system into soil;

(4) In the recharge process of adding nutrients and domesticated microorganisms, and injected $\mathrm{O}_{2}$

A typical example is a wood preservative contaminated groundwater, the main pollutants pentachlorophenol (PCP). The groundwater is pumped from pollution, adjusting the $\mathrm{pH}$ value and adding nutrients into the temperature controlled solid membrane bioreactor, PCP fully degraded inside[10].

\section{Conclusion}

At present, the groundwater pollution is very serious, directly and indirectly endanger human health, so it is imperative to explore an economical and effective measures for prevention and control of pollution. Bioremediation technology as a development potential, high efficiency and less investment in green environmental remediation technology, has attracted more and more attention.

The pollution to surface water is more complex and subtle. In the United States and Europe, bioremediation technology mainly in the laboratory and pilot phase, the practice has achieved initial results. In our country, some universities and research institutions also on groundwater pollution bioremediation were studied, but research in this aspect is not perfect, is still in the initial stage, 
only some small scale and pilot study, it is necessary to strengthen the research and application.

At the same time, during the course of the study, the bioremediation of groundwater pollution still exists some shortcomings and problems needing attention in many ways. For example, many organic pollutants in the underground is difficult to degrade, usually some microbial acclimation, the toxicity of pollutants sometimes inhibition of original microbial activity, injection well sometimes due to blocked by investment plus nutrients and oxygen would be excessive growth of microorganisms. Especially in situ biological repair of bioremediation, the organic matter how to react, they are the ability of biological degradation and biological degradation rate and environmental factors are not very clear. But because of its safety and maneuverability, bioremediation of groundwater pollution has wide application prospects in china.

\section{References}

[1]. Guimin Min, Guangliang Xu. Introduction to biotechnology[M]. China Environmental Science Press.2006.8.

[2]. Jing Li,Jiayao Zhang etc.,Bioremediation of contaminated groundwater[J]. Agriculture Environmental Protection,1997,16(6):283-285.

[3]. Jianwen Wang,Xianghua Wen.Modern environmental biotechnology[M].Beijing: Tsinghua University Press,2001.

[4]. Dezhong Shen.Bioremediation of environmental pollution[M].Beijing: Chemical Industry Press,2002.

[5]. Xuesong Yi,Liping Sun,Jian Yuan,etc,.Study on the adsorption of Pb2+ by peat in waste water[J]. Environmental Science and Management,2008,4（33）:123125.

[6]. Huiqiang Ma,Honglin Zhang ,Shuang Li,etc,.Heating Modification of Peat for Sorption of BTEX in Groundwater[J].Chemical and Biological Engineering,2010 (11): 72-75.

[7]. US Environmental Protection Agency. Analysis of selected enhancements for soil vapor extraction[M].Washington DC:National Service Center for Environmental Publications, 1997:3674.

[8]. Battelle Memorial Institute Columbus. Mult-i Site air sparging [M].Columbus: Environmental Security Technology Certification Program,2002:1572.

[9]. Richard M,Gersberg,et al. Variability in B TEX biodeg2 radation under denitrifying condition[J].W at. Res. 1995, 29 (2):545-550.

[10]. Guoqiang Huang,Xingang Li.The Evolution of In-situ Bioremediation for GroundwaterContaminated by Organic Chemicals[J].Progress in Chemistry, 2001 , (10):13-16. 